\title{
Positive solutions for a class of quasilinear Schrödinger equations with vanishing potentials
}

\section{Xiaonan Liu and Haibo Chen*}

\section{"Correspondence:}

math_chb@csu.edu.cn

School of Mathematics and

Statistics, Central South University,

Changsha, Hunan 410083, P.R. China

\section{Abstract}

In this paper, we study the following quasilinear Schrödinger equation:

$$
-\Delta u+V(x) u-\Delta\left(u^{2}\right) u=K(x) g(u) \text { in } \mathbb{R}^{N},
$$

where $N \geq 3, V$ is a nonnegative continuous function, which can vanish at infinity, and $g$ is a continuous function with a quasicritical growth. Under some appropriate conditions, we get the existence of a positive solution by combining the variational method with a Hardy-type inequality.

MSC: 35B38; 35G99

Keywords: quasilinear Schrödinger equation; positive solution; vanishing potentials; variational method

\section{Introduction and main results}

In this paper, we consider the existence of positive solutions for the following class of quasilinear Schrödinger equations:

$$
\left\{\begin{array}{l}
-\Delta u+V(x) u-\Delta\left(u^{2}\right) u=K(x) g(u) \quad \text { in } \mathbb{R}^{N}, \\
u \in D^{1,2}\left(\mathbb{R}^{N}\right),
\end{array}\right.
$$

for $N \geq 3$ and assuming that $V, K: \mathbb{R}^{N} \rightarrow \mathbb{R}$ and $g: \mathbb{R} \rightarrow \mathbb{R}$ are continuous functions with $V, K$ being nonnegative functions and $g$ having a quasicritical growth.

The solutions of equation (1.1) are related to the existence of standing wave solutions for the following Schrödinger equations:

$$
i z_{t}=-\Delta z+W(x) z-K(x) \rho\left(|z|^{2}\right) z-k \Delta\left(l\left(|z|^{2}\right)\right) l^{\prime}\left(|z|^{2}\right) z
$$

where $z: \mathbb{R} \times \mathbb{R}^{N} \rightarrow \mathbb{C}, W: \mathbb{R}^{N} \rightarrow \mathbb{R}$ is a given potential; $\rho, l: \mathbb{R}^{+} \rightarrow \mathbb{R}$ are real functions. Equations like (1.2) appear more naturally in mathematical physics and have been derived as models of several physical phenomena corresponding to various types of $l$. The case $l(s)=s$ was used for the superfluid film equation in plasma physics [1]. In the case $l(s)=$ 
$(1+s)^{1 / 2}$, equation (1.2) models the self-channeling of a high-power ultra short laser in matter [2]. Equation (1.2) also appears in plasma physics and fluid mechanics [3] and in dissipative quantum mechanics [4]. While this paper deals with the model where $l(s)=s$ and we put $z(t, x)=\exp (-i E t) u(x)$ into (1.2), we get the equation (1.1) with $V(x)=W(x)-E$.

Recently, there has appeared a broad literature treating the existence of solutions about a variety of Schrödinger equations [5-14]. But here we just introduce some different assumptions on $V(x)$ for the quasilinear Schrödinger equations. Alves, Wang, and Shen [15] investigated the following quasilinear Schrödinger equation:

$$
-\Delta u+V(x) u+\frac{k}{2}\left[\Delta\left(u^{2}\right)\right] u=l(u), \quad x \in \mathbb{R}^{N},
$$

where $N \geq 3, l: \mathbb{R} \rightarrow \mathbb{R}$ are continuous function, $k$ is a parameter. They assume that the potential $V: \mathbb{R}^{N} \rightarrow \mathbb{R}$ is continuous and satisfies:

$\left(V_{1}\right) V(x) \geq V(0)>0$, for all $x \in \mathbb{R}^{N}$.

$\left(V_{2}\right) \lim _{|x| \rightarrow \infty} V(x)=V_{\infty}$ and $V(x) \leq V_{\infty}$, for all $x \in \mathbb{R}^{N}$.

Under these two conditions, they combined the variational method with perturbation arguments to get a solution.

In [16], Fang and Szulkin obtained the multiplicity of solutions and made an assumption on $V(x)$ as follows:

$\left(V_{3}\right) V$ is continuous, 1-periodic in $x_{i}, 1 \leq i \leq N$, and there exists a constant $a_{0}>0$ such that $V(x) \geq a_{0}$ for all $x \in \mathbb{R}^{N}$.

In [17], the authors assumed $V(x)$ to satisfy

$\left(V_{4}\right) V \in C\left(\mathbb{R}^{N}, \mathbb{R}\right), 0 \leq V(x) \leq V_{\infty}:=\lim _{|x| \rightarrow \infty} V(x)<\infty, V_{\infty}>0$, and $V(x)<V_{\infty}$ somewhere.

$\left(V_{5}\right)$ There are positive constants $M, A$ and $m$ such that, for $|x| \geq M, V(x) \leq V_{\infty}-\frac{A}{1+|x|^{m}}$ and under these conditions, they obtained a ground state positive solution and a ground state sign-changing solution by the Nehari method. For more interesting results [18-22, 26].

But in this paper, what we are interested in is the zero mass case, which occurs with the potential $V$ vanishing at infinity, that is,

$$
\lim _{|x| \rightarrow+\infty} V(x)=0 \quad(\text { shortly } V(\infty)=0)
$$

In [23], Aires and Souto studied the zero mass case and the potential $V$ satisfies the following assumptions:

$\left(V_{0}\right) V(x) \geq 0$, for all $x \in \mathbb{R}^{N}$.

$\left(V_{\infty}\right) V(x) \leq V_{\infty}$, for all $x \in \mathbb{R}^{N}$.

$\left(V_{\Lambda}\right)$ There are $\Lambda>0$ and $R>1$ such that

$$
\frac{1}{R^{4}} \inf _{|x| \geq R}|x|^{4} V(x) \geq \Lambda .
$$

Under these conditions, they obtained the existence of solutions for the following equations:

$$
\left\{\begin{array}{l}
-\Delta u+V(x) u-\Delta\left(u^{2}\right) u=g(u) \quad \text { in } \mathbb{R}^{N}, \\
u \in D^{1,2}\left(\mathbb{R}^{N}\right) .
\end{array}\right.
$$


To the best of our knowledge, except for $[23,24]$ there is no paper dealing with the quasilinear Schrödinger equations (1.1) with vanishing potentials.

We are motivated by the above articles and especially by the work of Alves and Souto [25]. In the present paper, we consider the hypotheses on the potential $V$ :

$\left(V_{1}^{\prime}\right) V(x), K(x)>0, \forall x \in \mathbb{R}^{N}$ and $K \in L^{\infty}\left(\mathbb{R}^{N}\right)$.

$\left(V_{2}^{\prime}\right)$ If $A_{n} \subset \mathbb{R}^{N}$ is a sequence of Borel sets such that $\left|A_{n}\right| \leq R$, for all $n$ and some $R>0$, then

$$
\lim _{r \rightarrow+\infty} \int_{A_{n} \cap B_{r}^{c}(0)} K(x) d x=0, \quad \text { uniformly in } n \in \mathbb{N}
$$

$\left(V_{3}^{\prime}\right)$ One of the following conditions occurs:

$$
\left(K_{1}\right) \quad \frac{K}{V} \in L^{\infty}\left(\mathbb{R}^{N}\right)
$$

or there is $p \in\left(2,2^{*}\right)$ such that

$$
\left(K_{2}\right) \frac{K(x)}{[V(x)]^{\frac{2^{*}-p}{2^{*}-2}}} \rightarrow 0 \quad \text { as }|x| \rightarrow+\infty .
$$

In order to obtain our result, we also need to assume the following conditions on $g(x)$ $\left(g_{1}\right)$ :

$$
\limsup _{s \rightarrow 0} \frac{g(s)}{s}=0 \quad \text { if }\left(K_{1}\right) \text { holds, }
$$

or

$$
\limsup _{s \rightarrow 0^{+}} \frac{|g(s)|}{s^{p-1}}<+\infty \quad \text { if }\left(K_{2}\right) \text { holds. }
$$

$\left(g_{2}\right): g$ has a quasicritical growth, that is,

$$
\limsup _{s \rightarrow+\infty} \frac{g(s)}{s^{2^{*}-1}}<+\infty
$$

$\left(g_{3}\right)$ : there exists $\theta>2$ such that

$$
2 \theta G(s) \leq s g(s), \quad \forall s>0
$$

$\left(g_{4}\right)$ : there exists $v>\frac{1}{1-2\|K\|_{L} \infty}$ such that

$$
\frac{1}{2 v} g(s) s-G(s) \geq-V(x)|s|^{2}, \quad \forall s>0 .
$$

Remark 1.1 The condition $\left(g_{4}\right)$ is the key to prove the boundedness of the Cerami sequence. 
Remark 1.2 When $N=3$, a typical example that verifies $\left(g_{1}\right)-\left(g_{4}\right)$ is given by

$$
g(s)=c_{1} s|s|^{\kappa-1}
$$

where $\kappa+1 \geq \max \{2 \theta, 2 v\}$ and $p \leq \kappa+1 \leq 2^{*}$.

Now, we are able to state our main theorem.

Theorem 1.3 Suppose that $V, K$ satisfy $\left(V_{1}^{\prime}\right)-\left(V_{3}^{\prime}\right)$ and $g$ satisfies $\left(g_{1}\right)-\left(g_{4}\right)$. Then problem (1.1) possesses a positive solution.

The remainder of this paper is organized as follows. In Section 2, some preliminary results are presented. In Section 3, we prove the boundedness of the Cerami sequences. In Section 4, we give the proof of our main result.

\section{Preliminaries}

First, in this paper, we consider the Sobolev space

$$
E=\left\{u \in D^{1,2}\left(\mathbb{R}^{N}\right): \int_{\mathbb{R}^{N}} V(x)|u|^{2} d x<\infty\right\}
$$

endowed with the norm

$$
\|u\|_{E}=\left(\int_{\mathbb{R}^{N}}\left(|\nabla u|^{2}+V(x)|u|^{2}\right) d x\right)^{1 / 2} .
$$

It is well known that the embedding $D^{1,2} \hookrightarrow L^{2^{*}}\left(\mathbb{R}^{N}\right)$ is continuous, i.e. there exists $C>0$ such that

$$
\|u\|_{2^{*}} \leq C\|u\|_{D^{1,2}}, \quad \forall u \in D^{1,2} .
$$

Next, we establish the variational structure of problem (1.1). We observe that the natural energy functional associated with (1.1) is

$$
J(u)=\frac{1}{2} \int_{\mathbb{R}^{N}}\left(1+2 u^{2}\right)|\nabla u|^{2} d x+\frac{1}{2} \int_{\mathbb{R}^{N}} V(x) u^{2} d x-\int_{\mathbb{R}^{N}} K(x) G(u) d x,
$$

and it is obvious that the functional $J$ is not well defined in general, because $\int_{\mathbb{R}^{N}} u^{2}|\nabla u|^{2} d x$ is not finite, for all $u \in D^{1,2}\left(\mathbb{R}^{N}\right)$. In order to overcome this difficulty, we adopt the following method: a change of variables as introduced by Colin and Jeanjean in [27]: $v=f^{-1}(u)$, where $f$ is defined by

$$
f^{\prime}(t)=\frac{1}{\sqrt{1+2 f^{2}(t)}} \text { on }[0,+\infty) \quad \text { and } \quad f(t)=-f(-t) \quad \text { on }(-\infty, 0]
$$

and has the following properties.

Lemma 2.1 The function $f$ possesses the following properties:

(1) $f$ is uniquely defined, is $C^{\infty}$, and is invertible; 
(2) $\left|f^{\prime}(t)\right| \leq 1, \forall t \in \mathbb{R}$

(3) $|f(t)| \leq|t|, \forall t \in \mathbb{R}$;

(4) $\frac{f(t)}{t} \rightarrow 1$ as $t \rightarrow 0$;

(5) $\frac{f(t)}{\sqrt{t}} \rightarrow 2^{\frac{1}{4}}$ as $t \rightarrow+\infty$;

(6) $\frac{f(t)}{2} \leq t f^{\prime}(t) \leq f(t), \forall t \geq 0$;

(7) $|f(t)| \leq 2^{\frac{1}{4}}|t|^{\frac{1}{2}}, \forall t \in \mathbb{R}$;

(8) $\frac{f^{2}(t)}{2} \leq t f(t) f^{\prime}(t) \leq f^{2}(t), \forall t \in \mathbb{R}$;

(9) there exists a positive constant $C$ such that

$$
|f(t)| \geq \begin{cases}C|t| & \text { if }|t| \leq 1 \\ C|t|^{\frac{1}{2}} & \text { if }|t| \geq 1\end{cases}
$$

(10) $\left|f(t) f^{\prime}(t)\right| \leq \frac{1}{\sqrt{2}}, \forall t \in \mathbb{R}$.

After the change of variables, the functional $J(u)$ can be rewritten as follows:

$$
I(v)=J(f(v))=\frac{1}{2} \int_{\mathbb{R}^{N}}|\nabla v|^{2}+V(x)|f(v)|^{2} d x-\int_{\mathbb{R}^{N}} K(x) G(f(v)) d x,
$$

which is well defined on $E$ and is of $C^{1}$ class, and, for all $\omega \in E$, we have

$$
\left\langle I^{\prime}(v), \omega\right\rangle=\int_{\mathbb{R}^{N}} \nabla v \nabla \omega d x+\int_{\mathbb{R}^{N}} V(x) f(v) f^{\prime}(v) \omega d x-\int_{\mathbb{R}^{N}} K(x) g(f(v)) f^{\prime}(v) \omega d x .
$$

Remark 2.2 Note that the functional $\Psi: E \rightarrow \mathbb{R}$, given by

$$
\Psi(v)=\int_{\mathbb{R}^{N}}\left(|\nabla v|^{2}+V(x) f^{2}(v)\right) d x,
$$

is a $C^{1}$ function and

$$
\Psi(v) \leq\|v\|^{2}, \quad \text { for all } v \in E .
$$

Moreover, there is a constant $\beta>0$ such that

$$
\beta\|v\|^{2} \leq \Psi(v)+(\Psi(v))^{2^{*} / 2}, \quad \text { for all } v \in E .
$$

We note that the critical points of $I$ are weak solutions of the problem

$$
\begin{cases}-\Delta v+V(x) f(v) f^{\prime}(v)=K(x) g(f(v)) f^{\prime}(v) & \text { in } \mathbb{R}^{N}, \\ v \in D^{1,2}\left(\mathbb{R}^{N}\right), & v>0\end{cases}
$$

We also note that if $v \in C^{2}\left(\mathbb{R}^{N}\right) \cap D^{1,2}\left(\mathbb{R}^{N}\right)$ is a critical point of the functional $I$, then $u=f(v)$ is a classical solution of (1.1).

Remark 2.3 Since we intend to prove the existence of positive solutions of the problem (1.1), we can consider

$$
g(t)=0, \quad \forall t \leq 0
$$




\section{The boundedness of the Cerami sequences}

We say that a sequence $\left(v_{n}\right) \in E$ is called a Cerami sequence of $I$ at level $c$ if

$$
I\left(v_{n}\right) \rightarrow c \quad \text { and } \quad\left(1+\left\|v_{n}\right\|_{E}\right)\left\|I^{\prime}\left(v_{n}\right)\right\| \rightarrow 0 \quad \text { as } n \rightarrow \infty .
$$

The functional $I$ satisfies the Cerami condition if any Cerami sequence possesses a convergent subsequence in $E$.

Lemma 3.1 (Hardy-type inequality (see [25])) Assume that $V, K$ satisfy $\left(V_{1}^{\prime}\right)-\left(V_{3}^{\prime}\right)$, then $E$ is compactly embedded in $L_{k}^{q}\left(\mathbb{R}^{N}\right)$ for all $q \in\left(2,2^{*}\right)$ if $\left(K_{1}\right)$ holds. If $\left(K_{3}\right)$ holds, $E$ is compactly embedded in $L_{k}^{p}\left(\mathbb{R}^{N}\right)$, where

$$
L_{k}^{r}\left(\mathbb{R}^{N}\right)=\left\{v: \mathbb{R}^{N} \rightarrow \mathbb{R}: v \text { is measurable and } \int_{\mathbb{R}^{N}} K(x)|v|^{r}<\infty\right\} .
$$

Lemma 3.2 Suppose that $V, K$ satisfy $\left(V_{1}^{\prime}\right)-\left(V_{3}^{\prime}\right)$ and $g$ satisfies $\left(g_{1}\right),\left(g_{2}\right)$. Let $\left(v_{n}\right)$ be a sequence such that $v_{n} \rightarrow v$ in $E$. Then

$$
\lim _{n \rightarrow+\infty} \int_{\mathbb{R}^{N}} K(x) G\left(v_{n}\right) d x=\int_{\mathbb{R}^{N}} K(x) G(v) d x
$$

and

$$
\lim _{n \rightarrow+\infty} \int_{\mathbb{R}^{N}} K(x) g\left(v_{n}\right) v_{n} d x=\int_{\mathbb{R}^{N}} K(x) g(v) v d x .
$$

The proof follows from the idea of Lemma 2.2 in [25].

Lemma 3.3 Suppose $V, K$ satisfy $\left(V_{1}^{\prime}\right)-\left(V_{3}^{\prime}\right)$ and $g$ satisfies $\left(g_{1}\right)-\left(g_{4}\right)$, if $\left(v_{n}\right) \subset E$ is a Cerami sequence of $I$, then $\left(v_{n}\right)$ is bounded in $E$.

Proof Let $\left(v_{n}\right)$ be a Cerami sequence of $I$, then

$$
I\left(v_{n}\right) \rightarrow c \quad \text { and } \quad\left(1+\left\|v_{n}\right\|_{E}\right)\left\|I^{\prime}\left(v_{n}\right)\right\| \rightarrow 0 \quad \text { as } n \rightarrow \infty,
$$

that is,

$$
I\left(v_{n}\right)=\frac{1}{2} \int_{\mathbb{R}^{N}}\left(\left|\nabla v_{n}\right|^{2}+V(x) f^{2}\left(v_{n}\right)\right) d x-\int_{\mathbb{R}^{N}} K(x) G\left(f\left(v_{n}\right)\right) d x=c+o_{n}(1) .
$$

For $\omega \in E$, we have

$$
\left\langle I^{\prime}\left(v_{n}\right), \omega\right\rangle=\int_{\mathbb{R}^{N}} \nabla v_{n} \nabla \omega d x+\int_{\mathbb{R}^{N}} V(x) f\left(v_{n}\right) f^{\prime}\left(v_{n}\right) \omega d x-\int_{\mathbb{R}^{N}} K(x) g\left(f\left(v_{n}\right)\right) f^{\prime}\left(v_{n}\right) \omega d x .
$$

Taking $\omega=\omega_{n}=\frac{f\left(v_{n}\right)}{f^{\prime}\left(v_{n}\right)}$, by property $(6)$ of Lemma 2.1 , we have

$$
\left|\omega_{n}\right| \leq 2\left|v_{n}\right| \quad \text { and } \quad\left|\nabla \omega_{n}\right| \leq 2\left|\nabla v_{n}\right|
$$

and thus $\omega_{n} \in E$ and $\left\|\omega_{n}\right\| \leq 2\left\|v_{n}\right\|$. 
From (3.1), we obtain

$$
\left\langle I^{\prime}\left(v_{n}\right), \omega_{n}\right\rangle=o_{n}(1)
$$

that is,

$$
\Psi\left(v_{n}\right)+\int_{\mathbb{R}^{N}} \frac{2 f^{2}\left(v_{n}\right)}{1+2 f^{2}\left(v_{n}\right)}\left|\nabla v_{n}\right|^{2} d x-\int_{\mathbb{R}^{N}} K(x) g\left(f\left(v_{n}\right)\right) f\left(v_{n}\right) d x=o_{n}(1) .
$$

It follows from (3.2) and (3.3) that

$$
I\left(v_{n}\right)-\frac{1}{2 v}\left\langle I^{\prime}\left(v_{n}\right), \omega_{n}\right\rangle=c+o_{n}(1)
$$

and thus

$$
\left(\frac{1}{2}-\frac{1}{2 v}\right) \Psi\left(v_{n}\right)+\int_{\mathbb{R}^{N}}\left[\frac{1}{2 v} K(x) g\left(f\left(v_{n}\right)\right) f\left(v_{n}\right)-K(x) G\left(f\left(v_{n}\right)\right)\right] d x \leq c+o_{n}(1) .
$$

Combining (3.4) with $\left(g_{4}\right)$, we get

$$
\left(\frac{1}{2}-\frac{1}{2 v}\right) \Psi\left(v_{n}\right)-\int_{\mathbb{R}^{N}} K(x) V(x)\left|f\left(v_{n}\right)\right|^{2} d x \leq c+o_{n}(1),
$$

that is,

$$
\begin{aligned}
\left(\frac{1}{2}-\frac{1}{2 v}\right) \Psi\left(v_{n}\right) & \leq c+\int_{\mathbb{R}^{N}} K(x) V(x)\left|f\left(v_{n}\right)\right|^{2} d x+o_{n}(1) \\
& \leq c+\|K(x)\|_{L^{\infty}} \int_{\mathbb{R}^{N}} V(x)\left|f\left(v_{n}\right)\right|^{2} d x+o_{n}(1) \\
& \leq c+\|K(x)\|_{L^{\infty}} \Psi\left(v_{n}\right)+o_{n}(1)
\end{aligned}
$$

So, we have

$$
\left(\frac{1}{2}-\frac{1}{2 v}-\|K(x)\|_{L^{\infty}}\right) \Psi\left(v_{n}\right) \leq c+o_{n}(1)
$$

Therefore, $\Psi\left(v_{n}\right)$ is bounded, and consequently from Remark 2.2, the sequence $\left(v_{n}\right)$ is bounded in $E$.

Since the sequence $\left(v_{n}\right)$ given by Lemma 3.3 is bounded in $E$, there exist a constant $M>0$ and $v \in E$, and a subsequence of $\left(v_{n}\right)$, still denoted by $\left(v_{n}\right)$, such that $\left\|v_{n}\right\|_{E} \leq M,\|v\|_{E} \leq M$ and

$$
\begin{aligned}
& v_{n} \rightarrow v, \quad \text { weakly in } E, \quad v_{n} \rightarrow v \quad \text { in } L_{\text {loc }}^{p}\left(\mathbb{R}^{N}\right), p \in\left[1,2^{*}\right), \\
& v_{n}(x) \rightarrow v(x), \quad \text { a.e. in } \mathbb{R}^{N} .
\end{aligned}
$$

For concluding also the proof of Theorem 1.3, we need one more lemma. 
Lemma 3.4 Assume $V, K$ satisfy $\left(V_{1}^{\prime}\right)-\left(V_{3}^{\prime}\right)$, if the sequence $\left(v_{n}\right)$ satisfies $(C)$ sequence, then the following statements hold:

(i) For each $\epsilon>0$, there exists $r_{0} \geq 1$ such that, for $r \geq r_{0}$,

$$
\begin{aligned}
& \limsup _{n \rightarrow \infty} \int_{B_{2 r}^{c}}\left(\left|\nabla v_{n}\right|^{2}+V(x)\left|f\left(v_{n}\right)\right|^{2}\right) d x<\epsilon, \\
& \limsup _{n \rightarrow \infty} \int_{B_{2 r}^{c}}\left(\left|\nabla v_{n}\right|^{2}+V(x) f\left(v_{n}\right) f^{\prime}\left(v_{n}\right) v_{n}\right) d x<\epsilon,
\end{aligned}
$$

and

$$
\begin{aligned}
& \lim _{n \rightarrow \infty} \int_{\mathbb{R}^{N}} V(x)\left|f\left(v_{n}\right)\right|^{2} d x=\int_{\mathbb{R}^{N}} V(x)|f(v)|^{2} d x, \\
& \lim _{n \rightarrow \infty} \int_{\mathbb{R}^{N}} V(x) f\left(v_{n}\right) f^{\prime}\left(v_{n}\right) v_{n} d x=\int_{\mathbb{R}^{N}} V(x) f(v) f^{\prime}(v) v d x .
\end{aligned}
$$

(ii) The weak limit $v \in E$ is a critical point of the functional $I$.

(iii) $v_{n} \rightarrow v$ in E, i.e. the functional I satisfies the Cerami condition in E.

Proof The proof is almost similar to the one of Lemma 3.4 in [26], so we omit it here.

\section{Proof of main results}

In this section, we will give the proof of Theorem 1.3, which mainly relies on the following mountain-pass theorem.

Theorem 4.1 (see $[28,29]$ ) Let $X$ be a real Banach space and let $I \in C^{1}(X, \mathbb{R})$. Let $\Sigma$ be a closed subset of $X$ which disconnects (archwise) $X$ into distinct connected components $X_{1}$ and $X_{2}$. Suppose further that $I(0)=0$ and

$\left(I_{1}\right) 0 \in X_{1}$ and there is $\alpha>0$ such that $\left.I\right|_{\Sigma} \geq \alpha>0$,

$\left(I_{2}\right)$ there is $e \in X_{2}$ such that $I(e)<0$.

Then I possesses a $(c)_{c}$ sequence with $c \geq \alpha>0$ given by

$$
c=\inf _{\gamma \in \Gamma} \max _{t \in[0,1]} I(\gamma(t)),
$$

where

$$
\Gamma=\{\gamma \in C([0,1], X) ; \gamma(0)=0 \text { and } I(\gamma(1))<0\} .
$$

Proof of Theorem 1.3 Clearly, by Lemmas 3.1-3.3 in Section 3, the functional $I$ satisfies the Cerami condition. Next, we prove that $I$ satisfies $\left(I_{1}\right)$ and $\left(I_{2}\right)$ of Theorem 4.1.

First of all, we note that $I(0)=0$. Now, for every $\rho>0$, define

$$
\Sigma_{\rho}=\left\{v \in E ; \Psi(v)=\rho^{2}\right\},
$$

where $\Psi$ was defined as before. Since the functional $\Psi$ is continuous, it follows that $\Sigma_{\rho}$ is a closed subset which disconnects the space $E$. Using $\left(V_{1}^{\prime}\right),\left(g_{1}\right),\left(g_{2}\right)$, and the Sobolev 
embedding inequality, we have

$$
\begin{aligned}
\int_{\mathbb{R}^{N}} K(x) G(f(v)) & \leq\|K(x)\|_{L^{\infty}} \int_{\mathbb{R}^{N}} G(f(v)) d x \\
& \leq\|K(x)\|_{L^{\infty}} \int_{\mathbb{R}^{N}} \epsilon V(x)|f(v)|^{2}+C_{\epsilon}|f(v)|^{2^{*}} d x \\
& \leq\|K(x)\|_{L^{\infty}} \epsilon(\Psi(v))+\|K(x)\|_{L^{\infty}} C_{\epsilon} S\left(\int_{\mathbb{R}^{N}}|\nabla v|^{2} d x\right)^{\frac{2^{*}}{2}} \\
& \leq\|K(x)\|_{L^{\infty}} \epsilon \Psi(v)+\|K(x)\|_{L^{\infty}} C_{\epsilon} S(\Psi(v))^{\frac{2^{*}}{2}},
\end{aligned}
$$

from which it follows that

$$
I(v) \geq \frac{1}{2} \rho^{2}-\epsilon\|K(x)\|_{L^{\infty}} \rho^{2}-C_{\epsilon} S\|K(x)\|_{L^{\infty}} \rho^{2^{*}} .
$$

Since $2^{*}>2$, it implies that, for $\rho>0$ sufficiently small, there exists $\alpha>0$ such that

$$
I(v) \geq \alpha>0, \quad \forall v \in \Sigma_{\rho} .
$$

Thus, condition $\left(I_{1}\right)$ is satisfied.

Now, let us show that there is $\varphi \in E$ such that

$$
I(t \varphi) \rightarrow-\infty \quad \text { as } t \rightarrow+\infty
$$

Next we consider $\varphi \in C_{0}^{\infty}\left(\mathbb{R}^{N}\right)$ satisfying $\operatorname{supp}(\varphi)=\bar{B}_{1}$ and $0 \leq \varphi(x) \leq 1, \forall x \in B_{1}$. Indeed

$$
I(t \varphi)=\frac{1}{2} \int_{\mathbb{R}^{N}}|\nabla(t \varphi)|^{2}+V(x)|f(t \varphi)|^{2}-\int_{\mathbb{R}^{N}} K(x) G(f(t \varphi)) d x,
$$

by hypothesis $\left(g_{3}\right)$, there exist positive constants $C_{1}$ and $C_{2}$ such that

$$
G(t) \geq C_{1}|t|^{2 \theta}-C_{2}, \quad \forall t \in \mathbb{R},
$$

hence, it follows from the above inequality and property (3) of Lemma 2.1 that

$$
\begin{aligned}
I(t \varphi) & \leq \frac{t^{2}}{2} \int_{\mathbb{R}^{N}}|\nabla \varphi|^{2}+V(x)|\varphi|^{2} d x-\int_{B_{1}} K(x) G(f(t \varphi)) d x \\
& \leq \frac{t^{2}}{2} \int_{\mathbb{R}^{N}}|\nabla \varphi|^{2}+V(x)|\varphi|^{2} d x-\int_{B_{1}} C_{1} K(x)|f(t \varphi)|^{2 \theta}+C_{2} \int_{B_{1}} K(x) d x .
\end{aligned}
$$

Since the function $\frac{f(t)}{t}$ is decreasing for $t>0$ and

$$
0 \leq t \varphi(x) \leq t, \quad \forall x \in B_{1} \text { and } t>0,
$$

we have

$$
f(t) \varphi(x) \leq f(t \varphi(x)), \quad \forall x \in B_{1} \text { and } t>0,
$$


which together with (4.3) implies

$$
\begin{aligned}
I(t \varphi) \leq & t^{2}\left[\frac{1}{2} \int_{\mathbb{R}^{N}}\left(|\nabla \varphi|^{2}+V(x) \varphi^{2}\right) d x-C_{1} \frac{f^{2 \theta}(t)}{t^{2}} \int_{B_{1}}|\varphi|^{2 \theta} d x\right. \\
& \left.+\frac{C_{3}}{t^{2}} \int_{B_{1}} K(x) d x\right] .
\end{aligned}
$$

By properties (9) of Lemma 2.1 and provided that $\theta>2$, we conclude that

$$
\lim _{t \rightarrow+\infty} \frac{f^{2 \theta}(t)}{t^{2}}=+\infty
$$

and hence $\left(I_{2}\right)$ is satisfied.

Therefore, the existence of positive solution in $E$ for problem (2.6) follows from Theorem 4.1 and so $u=f(v)$ is a solution of problem (1.1). We finished the proof of Theorem 1.3.

\section{Competing interests}

The authors declare that they have no competing interests.

\section{Authors' contributions}

All authors carried out the proofs and the authors conceived of the study. All authors read and approved the final manuscript.

\section{Acknowledgements}

The authors thank the referees for valuable comments and suggestions which improved the presentation of this manuscript. This article was partially supported by Natural Science Foundation of China 11671403.

\section{Publisher's Note}

Springer Nature remains neutral with regard to jurisdictional claims in published maps and institutional affiliations.

Received: 19 December 2016 Accepted: 7 March 2017 Published online: 15 March 2017

\section{References}

1. Kurihura, S: Large-amplitude quasi-solitons in superfluids films. J. Phys. Soc. Jpn. 50, 3262-3267 (1981)

2. Ritchie, B: Relativistic self-focusing and channel formation in laser-plasma interactions. Phys. Rev. E. 50, 687-689 (1994)

3. Bass, F, Nasanov, NN: Nonlinear electromagnetic-spin waves. Phys. Rep. 189, 165-223 (1990)

4. Hasse, RW: A general method for the solution of nonlinear soliton and kink Schrödinger equation. Z. Phys. B. 37, 83-87 (1980)

5. Liu, $\mathrm{H}$, Chen, $\mathrm{H}$, Yang, X: Least energy sign-changing solutions for nonlinear Schrödinger equations with indefinite-sign and vanishing potential. Appl. Math. Lett. 53, 100-106 (2016)

6. Liu, H, Chen, H, Xiao, Q: Positive ground state solutions for a class of Schrödinger-Poisson systems with sign-changing and vanishing potential. Math. Methods Appl. Sci. (2016). doi:10.1002/mma.4110

7. Liu, H, Chen, H, Wang, G: Multiplicity for a 4-sublinear Schrödinger-Poisson system with sign-changing potential via Morse theory. C. R. Math. 1(354), 75-80 (2016)

8. Liu, H, Chen, H: Multiple solutions for a nonlinear Schrödinger-Poisson system with sign-changing potential. Comput. Math. Appl. 71, 1405-1416 (2016)

9. Liu, H, Chen, H, Yang, X: Multiple solutions for superlinear Schrödinger-Poisson systems with sign-changing potential and nonlinearity. Comput. Math. Appl. 68(12), 1982-1990 (2014)

10. Sun, J, Chen, H, Nieto, J: On ground state solutions for some non-autonomous Schrödinger-Poisson systems. J. Differ. Equ. 252, 3365-3380 (2012)

11. Sun, M, Su, J, Zhao, L: Solutions of a Schrödinger-Poisson system with combined nonlinearities. J. Math. Anal. Appl. 442, 385-403 (2016)

12. Repovs, D: Stationary waves of Schrödinger-type equations with variable exponent. Anal. Appl. 13(6), 645-661 (2015)

13. Repovs, D, Zhang, B, Zhang, X: Existence and symmetry of solutions for critical fractional Schrödinger equations with bounded potentials. Nonlinear Anal. 142, 48-68 (2016)

14. Zhang, X, Zhang, B, Xiang, M: Ground states for fractional Schrödinger equations involving a critical nonlinearity. Adv. Nonlinear Anal. 5(3), 293-314 (2016)

15. Alves, CO, Wang, Y, Shen, Y: Soliton solutions for a class of quasilinear Schrödinger equations with a parameter. J. Differ. Equ. 259, 318-343 (2015)

16. Fang, X, Szulkin, A: Multiple solutions for a quasilinear Schrödinger equation. J. Differ. Equ. 254, 2015-2032 (2013) 
17. Liu, X, Liu, J, Wang, Z: Ground states for quasilinear Schrödinger equations with critical growth. Calc. Var. 46, 641-669 (2013)

18. Liu, J, Wang, Y, Wang, Z: Soliton solutions for quasilinear Schrödinger equations II. J. Differ. Equ. 187, 473-493 (2003)

19. Liu, J, Wang, Z: Soliton solutions for quasilinear Schrödinger equations. Proc. Am. Math. Soc. 131, 441-448 (2003)

20. Shi, H, Chen, H: Generalized quasilinear asymptotically periodic Schrödinger equations with critical growth. Comput. Math. Appl. 71, 849-858 (2016)

21. Moameni, A: Existence of soliton solution for a quasilinear Schrodinger equation involving critical exponent in $\mathbb{R}^{N}$. J. Differ. Equ. 229, 570-587 (2006)

22. Wu, X: Multiple solutions for quasilinear Schrödinger equations with a parameter. J. Differ. Equ. 256, 2619-2632 (2014)

23. Aires, JFL, Souto, MAS: Existence of solutions for a quasilinear Schrödinger equation with vanishing potentials. J. Math. Anal. Appl. 416, 924-946 (2014)

24. Shi, $\mathrm{H}$, Chen, $\mathrm{H}$ : Positive solutions for generalized quasilinear Schrödinger equations with potential vanishing at infinity. Appl. Math. Lett. 61, 137-142 (2016)

25. Alves, CO, Souto, MAS: Existence of solutions for a class of nonlinear Schrödinger equations with potential vanishing at infinity. J. Differ. Equ. 254, 1977-1991 (2013)

26. Song, H, Chen, C, Yan, Q: Infinitely many solutions for quasilinear Schrödinger equation with critical exponential growth in $\mathbb{R}^{N}$. J. Math. Anal. Appl. 439, 575-593 (2016)

27. Colin, M, Jeanjean, L: Solutions for a quasilinear Schrödinger equation: a dual approach. Nonlinear Anal. 56, 213-226 (2004)

28. Silva, EAB, Vieira, GF: Quasilinear asymptotically periodic Schrödinger equations with subcritical growth. Nonlinear Anal. 72, 2935-2949 (2010)

29. Silva, EAB, Vieira, GF: Quasilinear asymptotically periodic Schrödinger equations with critical growth. Calc. Var. 39, $1-33(2010)$

\section{Submit your manuscript to a SpringerOpen ${ }^{\circ}$ journal and benefit from:}

- Convenient online submission

- Rigorous peer review

Immediate publication on acceptance

- Open access: articles freely available online

- High visibility within the field

- Retaining the copyright to your article 